\title{
On Partial Sum of Tribonacci Numbers
}

\author{
Eunmi Choi and Jiin Jo \\ Department of Mathematics, Hannam University, Daejeon 306-791, Republic of Korea
}

Correspondence should be addressed to Eunmi Choi; emc@hnu.kr

Received 30 March 2015; Accepted 28 May 2015

Academic Editor: Nawab Hussain

Copyright (C) 2015 E. Choi and J. Jo. This is an open access article distributed under the Creative Commons Attribution License, which permits unrestricted use, distribution, and reproduction in any medium, provided the original work is properly cited.

We study the sum $s_{t}^{(k, r)}=\sum_{i=0}^{t} T_{k i+r}$ of $k$ step apart Tribonacci numbers for any $1 \leq r \leq k$. We prove that $s_{t}^{(k, r)}$ satisfies certain Tribonacci rule $s_{t}^{(k, r)}=a_{k} s_{t-1}^{(k, r)}+b_{k} s_{t-2}^{(k, r)}+s_{t-3}^{(k, r)}+\lambda$ with integers $a_{k}, b_{k}, c_{k}$, and $\lambda$.

\section{Introduction}

A Tribonacci sequence $\left\{T_{n}\right\}_{n \geq 0}$, which is a generalized Fibonacci sequence $\left\{F_{n}\right\}$, is defined by the Tribonacci rule $T_{n}=T_{n-1}+T_{n-2}+T_{n-3}$ with $T_{2}=T_{1}=1$ and $T_{0}=0$. The sequence can be extended to negative subscript $n$; hence few terms of the sequence are $\ldots,-3,2,0,-1,1,0,0,1,1,2,4,7, \ldots$. Each term in $\left\{T_{n}\right\}$ is called the Tribonacci number.

The sum of Fibonacci numbers is well expressed by $\sum_{i=0}^{n} F_{i}=F_{n+2}-1$, and moreover the sum of reciprocal Fibonacci numbers was studied intensively in [1-3]. For the sum $S_{n}=\sum_{i=0}^{n} T_{i}$ of Tribonacci numbers, there are some researches including [4-7]. In particular Kilic [6] proved the identity $S_{n}=(1 / 2)\left(T_{n+2}+T_{n}-1\right)$ by means of generating matrix calculations. And Irmak and Alp [5] proved an identity about the $k$ subscripted Tribonacci sum $S_{n, k}=$ $\sum_{i=0}^{n} T_{k i}$ using the three roots of $x^{3}-x^{2}-x-1=0$.

This paper is devoted to studying the sum of Tribonacci numbers as well as the sum $\sum_{i=0}^{t} T_{k i+r}$ of $k$ step apart Tribonacci numbers for any $1 \leq r \leq k$. Our method here is to employ the $k$-step Tribonacci rule $T_{n}=a_{k} T_{n-k}+$ $b_{k} T_{n-2 k}+c_{k} T_{n-3 k}\left(a_{k}, b_{k}, c_{k} \in \mathbb{Z}\right)$ that is a linear combination of $k$ distance Tribonacci numbers in [8]. For this purpose we will display all Tribonacci numbers in rectangle form with $k$ columns, called the $k$-tribo table:

$$
\begin{array}{cccccc}
T_{1} & T_{2} & \cdots & T_{r} & \cdots & T_{k} \\
T_{k+1} & T_{k+2} & \cdots & T_{k+r} & \cdots & T_{2 k} \\
T_{2 k+1} & T_{2 k+2} & \cdots & T_{2 k+r} & \ddots &
\end{array}
$$

Then $\sum_{i=0}^{t} T_{k i+r}$ can be regarded as a partial sum of $t+1$ entries in $r$ th column of the table. We denote it by $s_{t}^{(k, r)}$ for $1 \leq r \leq k$.

\section{Tribonacci Tables}

$T_{n}$ satisfies a Tribonacci rule $a_{1} T_{n-1}+b_{1} T_{n-2}+c_{1} T_{n-3}$ with $\left(a_{1}, b_{1}, c_{1}\right)=(1,1,1)$ and a 2 -step Tribonacci rule $a_{2} T_{n-2}+$ $b_{2} T_{n-4}+c_{2} T_{n-6}$ with $\left(a_{2}, b_{2}, c_{2}\right)=(3,1,1)$. Moreover the $k$ step Tribonacci rules for $T_{n}$ were proved in [8].

Lemma 1 (see [8]). Consider $T_{n}=a_{k} T_{n-k}+b_{k} T_{n-2 k}+T_{n-3 k}$ with $a_{k}=3 T_{k}-T_{k-6}$ and $b_{k}=-a_{-k}$ for any $1 \leq k<n$. The $\left\{a_{k}\right\}$ and $\left\{b_{k}\right\}$ satisfy $a_{k+3}=a_{k+2}+a_{k+1}+a_{k}$ and $b_{k}=b_{k+1}+b_{k+2}+b_{k+3}$ with $a_{1}=1, a_{2}=3, a_{3}=7$, and $b_{1}=b_{2}=1, b_{3}=-5$.

The recurrence $a_{k+3}=a_{k+2}+a_{k+1}+a_{k}$ implies that $\left\{a_{k}\right\}$ is a Tribonacci type sequence. By extending the subscript $k$ to negative integers, we have $a_{0}=3, a_{-1}=-1, a_{-2}=-1$, and so forth; thus $\left\{a_{k}\right\}=\{\ldots,-1,-5,5,-1,-1,3,1,3,7,11, \ldots\}$. In particular if $1 \leq k \leq 10$ then the coefficients $\left(a_{k}, b_{k}\right)$ are

\begin{tabular}{cc}
$k$ & $\left(a_{k}, b_{k}\right)$ \\
\hline 1 & $(1,1)$ \\
2 & $(3,1)$ \\
3 & $(7,-5)$ \\
4 & $(11,5)$ \\
5 & $(21,1)$ \\
6 & $(39,-11)$
\end{tabular}




$$
\begin{array}{cc}
7 & (71,15) \\
8 & (131,-3) \\
9 & (241,-23) \\
10 & (443,41)
\end{array}
$$

For example, $T_{31}$ is expressed by the 10-step Tribonacci rule $T_{31}=443 T_{21}+41 T_{11}+T_{1}=443(121415)+41(274)+1=$ 53798080. It shows that $T_{31}$ is a combination of three entries at 1st column of 10-tribo table. Similarly, by taking $k=11$, we have $a_{11}=815$ and $b_{11}=-21$, so $T_{31}=815 T_{20}-21 T_{9}+$ $T_{-2}=53798080$ which is a combination of three entries at 9th column of 11-tribo table.

Thus if $n=k t+r(0 \leq t, 1 \leq r \leq k)$ then $T_{n}$ is located at $(t+1)$ th row and $r$ th column in the $k$-tribo table and is a combination of three entries at $t, t-1$, and $(t-2)$ th row of $r$ th column. Now for the partial sum $s_{t}^{(k, r)}=\sum_{i=0}^{t} T_{k i+r}$ of $r$ th column in $k$-tribo table, let us begin with $k=3$ and 4 .

Theorem 2. When $k=3$ or 4 , the partial sum $s_{t}^{(k, r)}$ holds as follows:

$$
\begin{aligned}
& s_{t}^{(3, r)}= \begin{cases}7 s_{t-1}^{(3, r)}-5 s_{t-2}^{(3, r)}+s_{t-3}^{(3, r)}-1 & \text { if } r=1 \\
7 s_{t-1}^{(3, r)}-5 s_{t-2}^{(3, r)}+s_{t-3}^{(3, r)}+1 & \text { if } r \neq 1\end{cases} \\
& = \begin{cases}\frac{1}{T_{3}}\left(T_{3(t+1)+r}-4 T_{3 t+r}+T_{3(t-1)+r}+1\right) & \text { if } r=1 \\
\frac{1}{T_{3}}\left(T_{3(t+1)+r}-4 T_{3 t+r}+T_{3(t-1)+r}-1\right) & \text { if } r \neq 1\end{cases}
\end{aligned}
$$

(2)

$$
\begin{aligned}
& s_{t}^{(4, r)}=\left\{\begin{array}{rr}
11 s_{t-1}^{(4, r)}+5 s_{t-2}^{(4, r)}+s_{t-3}^{(4, r)}-4 & \text { if } r=1 \\
11 s_{t-1}^{(4, r)}+5 s_{t-2}^{(4, r)}+s_{t-3}^{(4, r)}+4 & \text { if } r \neq 1
\end{array}\right. \\
& = \begin{cases}\frac{1}{T_{4}^{2}}\left(T_{4(t+1)+r}+6 T_{4 t+r}+T_{4(t-1)+r}+4\right) & \text { if } r=1 \\
\frac{1}{T_{4}^{2}}\left(T_{4(t+1)+r}+6 T_{4 t+r}+T_{4(t-1)+r}-4\right) & \text { if } r \neq 1\end{cases}
\end{aligned}
$$

for $t \geq 1$.

Proof. The 3-tribo table produces a table of $s_{t}^{(3, r)}$ as follows:

\begin{tabular}{ccc} 
3-tribo & table \\
\hline 1 & 1 & 2 \\
4 & 7 & 13 \\
24 & 44 & 81 \\
149 & 274 & 504 \\
927 & 1705 & $\ldots$
\end{tabular}

\begin{tabular}{c|ccc}
$t$ & $s_{t}^{(3,1)}$ & $s_{t}^{(3,2)}$ & $s_{t}^{(3,3)}$ \\
\hline 0 & 1 & 1 & 2 \\
1 & 5 & 8 & 15 \\
2 & 29 & 52 & 96 \\
3 & 178 & 326 & 600 \\
4 & 1105 & 2031 & 3736
\end{tabular}

If $t=4$ then it can be observed that

$$
\begin{aligned}
s_{4}^{(3,1)} & =1105=7(178)-5(29)+5-1 \\
& =7 s_{3}^{(3,1)}-5 s_{2}^{(3,1)}+s_{1}^{(3,1)}-1, \\
s_{4}^{(3,2)} & =2031=7(326)-5(52)+8+1 \\
& =7 s_{3}^{(3,2)}-5 s_{2}^{(3,2)}+s_{1}^{(3,2)}+1, \\
s_{4}^{(3,3)} & =3736=7(600)-5(96)+15+1 \\
& =7 s_{3}^{(3,3)}-5 s_{2}^{(3,3)}+s_{1}^{(3,3)}+1 .
\end{aligned}
$$

For some $t>4$, we assume $s_{t}^{(3, r)}=7 s_{t-1}^{(3, r)}-5 s_{t-2}^{(3, r)}+s_{t-3}^{(3, r)} \pm 1$ with minus sign if $r=1$, otherwise plus sign. Then the next partial sum $s_{t+1}^{(3, r)}$ satisfies

$$
\begin{aligned}
s_{t+1}^{(3, r)}= & s_{t}^{(3, r)}+T_{3(t+1)+r} \\
= & \left(7 s_{t-1}^{(3, r)}-5 s_{t-2}^{(3, r)}+s_{t-3}^{(3, r)} \pm 1\right) \\
& +\left(7 T_{3 t+r}-5 T_{3(t-1)+r}+T_{3(t-2)+r}\right) \\
= & 7 s_{t}^{(3, r)}-5 s_{t-1}^{(3, r)}+s_{t-2}^{(3, r)} \pm 1
\end{aligned}
$$

due to Lemma 1. And we also notice that

$$
\begin{aligned}
T_{10}-4 T_{7}+T_{4}+1 & =149-4(24)+4+1=2(29) \\
& =2 s_{2}^{(3,1)}=T_{3} s_{2}^{(3,1)}, \\
T_{11}-4 T_{8}+T_{5}-1 & =274-4(44)+7-1=2(52) \\
& =2 s_{2}^{(3,2)}=T_{3} s_{2}^{(3,2)}, \\
T_{12}-4 T_{9}+T_{6}-1 & =504-4(81)+13-1=2(96) \\
& =2 s_{2}^{(3,3)}=T_{3} s_{2}^{(3,3)},
\end{aligned}
$$

so it shows $T_{3} s_{2}^{(3, r)}=T_{3(3)+r}-4 T_{3(2)+r}+T_{3+r} \pm 1$ with minus sign if $r=1$, otherwise plus. Hence if we assume 
$T_{3} s_{t}^{(3, r)}=T_{3(t+1)+r}-4 T_{3 t+r}+T_{3(t-1)+r} \pm 1$ for some $t$ then it follows from Lemma 1 that

$$
\begin{aligned}
T_{3} s_{t+1}^{(3, r)}= & T_{3}\left(s_{t}^{(3, r)}+T_{3(t+1)+r}\right) \\
= & T_{3(t+1)+r}-4 T_{3 t+r}+T_{3(t-1)+r} \pm 1+T_{3} T_{3(t+1)+r} \\
= & \left(7 T_{3(t+1)+r}-5 T_{3 t+r}+T_{3(t-1)+r}\right)-4 T_{3(t+1)+r} \\
& +T_{3 t+r} \pm 1 \\
= & T_{3(t+2)+r}-4 T_{3(t+1)+r}+T_{3 t+r} \pm 1 .
\end{aligned}
$$

Similarly the 4-tribo table makes the table of $s_{t}^{(4, r)}$ :

\begin{tabular}{cccc}
\multicolumn{4}{c}{ 4-tribo table } \\
\hline 1 & 1 & 2 & 4 \\
7 & 13 & 24 & 44 \\
81 & 149 & 274 & 504 \\
927 & 1705 & 3136 & 5768
\end{tabular}

\begin{tabular}{c|cccc}
$t$ & $s_{t}^{(4,1)}$ & $s_{t}^{(4,2)}$ & $s_{t}^{(4,3)}$ & $s_{t}^{(4,4)}$ \\
\hline 0 & 1 & 1 & 2 & 4 \\
1 & 8 & 14 & 26 & 48 \\
2 & 89 & 163 & 300 & 552 \\
3 & 1016 & 1868 & 3436 & 6320
\end{tabular}

When $t=3$, experimental observations show that

$$
\begin{aligned}
s_{3}^{(4,1)} & =1016=(11) 89+(5) 8+1-4 \\
& =11 s_{2}^{(4,1)}+5 s_{1}^{(4,1)}+s_{0}^{(4,1)}-4, \\
s_{3}^{(4,2)} & =1868=11(163)+5(14)+5 \\
& =11 s_{2}^{(4,2)}+5 s_{1}^{(4,2)}+s_{0}^{(4,2)}+4,
\end{aligned}
$$

and so on. Hence if we assume $s_{t}^{(4, r)}=11 s_{t-1}^{(4, r)}+5 s_{t-2}^{(4, r)}+s_{t-3}^{(4, r)} \pm 4$ with - sign if $r=1$, otherwise + sign for some $t>3$, then $s_{t+1}^{(4, r)}$ is equal to

$$
\begin{aligned}
s_{t+1}^{(4, r)}= & s_{t}^{(4, r)}+T_{4(t+1)+r} \\
= & \left(11 s_{t-1}^{(4, r)}+5 s_{t-2}^{(4, r)}+s_{t-3}^{(4, r)} \pm 4\right) \\
& +\left(11 T_{4 t+r}+5 T_{4(t-1)+r}+T_{4(t-2)+r}\right) \\
= & 11 s_{t}^{(4, r)}+5 s_{t-1}^{(4, r)}+s_{t-2}^{(4, r)} \pm 4 .
\end{aligned}
$$

On the other hand, it is easy to see that

$$
\begin{aligned}
T_{17} & +6 T_{13}+T_{9}+T_{4}=10609+6(927)+81+4 \\
& =4^{2}(1016)=T_{4}^{2} s_{3}^{(4,1)}, \\
T_{18} & +6 T_{14}+T_{10}-T_{4}=19513+6(1705)+149-4 \\
& =4^{2}(1868)=T_{4}^{2} s_{3}^{(4,2)}, \\
\vdots & \\
T_{20} & +6 T_{16}+T_{12}-T_{4}=66012+6(5768)+504-4 \\
& =4^{2}(6320)=T_{4}^{2} s_{3}^{(4,4)} .
\end{aligned}
$$

So by assuming $T_{4}^{2} s_{t-1}^{(4, r)}=T_{4 t+r}+6 T_{4(t-1)+r}+T_{4(t-2)+r} \pm T_{4}$ for some $t$, we have

$$
\begin{aligned}
T_{4(t+1)+r}+6 T_{4 t+r}+T_{4(t-1)+r} \pm T_{4} \\
=\left(11 T_{4 t+r}+5 T_{4(t-1)+r}+T_{4(t-2)+r}\right)+6 T_{4 t+r} \\
\quad+T_{4(t-1)+r} \pm T_{4} \\
=\left(T_{4 t+r}+6 T_{4(t-1)+r}+T_{4(t-2)+r} \pm T_{4}\right)+16 T_{4 t+r} \\
=T_{4}^{2} s_{t-1}^{(4, r)}+T_{4}^{2} T_{4 t+r}=T_{4}^{2}\left(s_{t-1}^{(4, r)}+T_{4 t+r}\right)=T_{4}^{2} s_{t}^{(4, r)} .
\end{aligned}
$$

We remark that if $r=4$ in Theorem 2 then we have

$$
\begin{gathered}
\frac{1}{T_{4}^{2}}\left(T_{4(t+2)}+6 T_{4(t+1)}+T_{4 t}-4\right) \\
=s_{t}^{(4,4)}=\sum_{i=0}^{t} T_{4 i+4}=\sum_{i=0}^{t+1} T_{4 i} .
\end{gathered}
$$

Since $T_{4}=4$, our result is equal to Theorem 5 in [6] which was proven by means of generating matrix.

The expression of $s_{t}^{(4, r)}$ has tails \pm 4 according to $r=1$ or not and equals $T_{4(t+1)+r}+6 T_{4 t+r}+T_{4(t-1)+r} \pm 4$ multiplied by $1 / T_{4}^{2}$. Similarly $s_{t}^{(3, r)}$ has tails \pm 1 according to $r=1$ or not and equals $T_{3(t+1)+r}-4 T_{3 t+r}+T_{3(t-1)+r} \pm 1$ multiplied by $1 / T_{3}$. However, when $k \geq 5$ the expressions of $s_{t}^{(k, r)}$ quite differ from the case of $k=3,4$.

Theorem 3. Let $\left\{\lambda^{(r)}\right\}_{r=1}^{5}=\{-7,3,5,1,9\}$ and $\left\{\lambda^{(r)}\right\}_{r=1}^{6}=$ $\{-12,6,4,-2,8,10\}$. Then one has the following:

(1)

$$
\begin{aligned}
s_{t}^{(5, r)} & =21 s_{t-1}^{(5, r)}+s_{t-2}^{(5, r)}+s_{t-3}^{(5, r)}+\lambda^{(r)} \quad \text { for } t \geq 3 \\
& =\frac{1}{22}\left(T_{5(t+1)+r}+2 T_{5 t+r}+T_{5(t-1)+r}-\lambda^{(r)}\right)
\end{aligned}
$$


(2)

$$
\begin{aligned}
s_{t}^{(6, r)} & =39 s_{t-1}^{(6, r)}-11 s_{t-2}^{(6, r)}+s_{t-3}^{(6, r)}+\lambda^{(r)} \quad \text { for } t \geq 3 \\
& =\frac{1}{28}\left(T_{6(t+1)+r}-10 T_{6 t+r}+T_{6(t-1)+r}-\lambda^{(r)}\right)
\end{aligned}
$$

for $t \geq 1$.

Proof. The 5-tribo table yields a table of $s_{t}^{(5, r)}$ such that

\begin{tabular}{c|ccccc}
$t$ & $s_{t}^{(5,1)}$ & $s_{t}^{(5,2)}$ & $s_{t}^{(5,3)}$ & $s_{t}^{(5,4)}$ & $s_{t}^{(5,5)}$ \\
\hline 0 & 1 & 1 & 2 & 4 & 7 \\
1 & 14 & 25 & 46 & 85 & 156 \\
2 & 288 & 529 & 973 & 1790 & 3292 \\
3 & 6056 & 11138 & 20486 & 37680 & $\ldots$
\end{tabular}

Then by inspecting the table, we find that

$$
\begin{gathered}
s_{3}^{(5,1)}=21(288)+14+1-7=21 s_{2}^{(5,1)}+s_{1}^{(5,1)}+s_{0}^{(5,1)} \\
-7, \\
s_{3}^{(5,2)}=21(529)+25+1+3=21 s_{2}^{(5,2)}+s_{1}^{(5,2)}+s_{0}^{(5,2)} \\
+3, \\
\vdots \\
s_{3}^{(5,5)}=21(3292)+156+7+9=21 s_{2}^{(5,5)}+s_{1}^{(5,5)} \\
+s_{0}^{(5,5)}+9,
\end{gathered}
$$

so we have $s_{3}^{(t, r)}=21 s_{2}^{(t, r)}+s_{1}^{(t, r)}+s_{t-3}^{(t, r)}+\lambda^{(r)}$ for $t=5$ and $1 \leq r \leq t$. Now if we assume the identity is true for some $t$, then the $t+1$ partial sum follows

$$
\begin{aligned}
s_{t+1}^{(5, r)}= & s_{t}^{(5, r)}+T_{5(t+1)+r} \\
= & \left(21 s_{t-1}^{(5, r)}+s_{t-2}^{(5, r)}+s_{t-3}^{(5, r)}+\lambda^{(r)}\right) \\
& +\left(21 T_{5 t+r}+T_{5(t-1)+r}+T_{5(t-2)+r}\right) \\
= & 21 s_{t}^{(5, r)}+s_{t-1}^{(5, r)}+s_{t-2}^{(5, r)}+\lambda^{(r)}
\end{aligned}
$$

from Lemma 1. Moreover we notice that

$$
\begin{aligned}
& T_{21}+2 T_{16}+T_{11}+7=121415+2(5768)+274+7 \\
& =22(6056)=22 s_{3}^{(5,1)}, \\
& T_{22}+2 T_{17}+T_{12}-3=223317+2(10609)+504-3 \\
& =22(11138)=22 s_{3}^{(5,2)}, \\
& \vdots \\
& T_{25}+2 T_{20}+T_{15}-9=1389537+2(66012)+3136 \\
& -9=22 s_{3}^{(5,5)},
\end{aligned}
$$

so we have $T_{5(4)+r}+2 T_{5(3)+r}+T_{5(2)+r}-\lambda^{(r)}=22 s_{3}^{(5, r)}$. Hence, by assuming $22 s_{t}^{(5, r)}=T_{5(t+1)+r}+2 T_{5 t+r}+T_{5(t-1)+r}-\lambda^{(r)}$ for some $t$, we have

$$
\begin{aligned}
22 s_{t+1}^{(5, r)}= & \left(T_{5(t+1)+r}+2 T_{5 t+r}+T_{5(t-1)+r}-\lambda^{(r)}\right) \\
& +22 T_{5(t+1)+r} \\
= & \left(21 T_{5(t+1)+r}+T_{5 t+r}+T_{5(t-1)+r}\right) \\
& +\left(2 T_{5(t+1)+r}+T_{5 t+r}-\lambda^{(r)}\right) \\
= & T_{5(t+2)+r}+2 T_{5(t+1)+r}+T_{5 t+r}-\lambda^{(r)} .
\end{aligned}
$$

Now, for (2), we construct the table of $s_{t}^{(6, r)}$ from 6-tribo table:

\begin{tabular}{c|cccccc}
$t$ & $s_{t}^{(6,1)}$ & $s_{t}^{(6,2)}$ & $s_{t}^{(6,3)}$ & $s_{t}^{(6,4)}$ & $s_{t}^{(6,5)}$ & $s_{t}^{(6,6)}$ \\
\hline 0 & 1 & 1 & 2 & 4 & 7 & 13 \\
1 & 25 & 45 & 83 & 153 & 281 & 517 \\
2 & 952 & 1750 & 3219 & 5921 & 10890 & 20030 \\
3 & 36842 & 67762 & 124634 & 229238 & 421634 & $\ldots$
\end{tabular}

It is not hard to observe

$$
\begin{aligned}
& s_{3}^{(6,1)}=39(952)-11(25)+1-12=39 s_{2}^{(6,1)} \\
& -11 s_{1}^{(6,1)}+s_{0}^{(6,1)}-12, \\
& s_{3}^{(6,2)}=39(1750)-11(45)+1+6=39 s_{2}^{(6,2)} \\
& -11 s_{1}^{(6,2)}+s_{0}^{(6,2)}+6, \\
& \vdots \\
& s_{3}^{(6,6)}=39(20030)-11(517)+13+10=39 s_{2}^{(6,6)} \\
& -11 s_{1}^{(6,6)}+s_{0}^{(6,6)}+10,
\end{aligned}
$$

so it proves $s_{t}^{(6, r)}=39 s_{t-1}^{(6, r)}-11 s_{t-2}^{(6, r)}+s_{t-3}^{(6, r)}+\lambda^{(r)}$ when $t=3$. Thus if we assume the identity holds for some $t$ then Lemma 1 shows that

$$
\begin{aligned}
s_{t+1}^{(6, r)}= & s_{t}^{(6, r)}+T_{6(t+1)+r} \\
= & \left(39 s_{t-1}^{(6, r)}-11 s_{t-2}^{(6, r)}+s_{t-3}^{(6, r)}+\lambda^{(r)}\right) \\
& +\left(39 T_{6 t+r}-11 T_{6(t-1)+r}+T_{6(t-2)+r}\right) \\
= & 39 s_{t}^{(6, r)}-11 s_{t-1}^{(6, r)}+s_{t-2}^{(6, r)}+\lambda^{(r)} .
\end{aligned}
$$


Finally we also notice $28 s_{2}^{(6, r)}=T_{6(3)+r}-10 T_{6(2)+r}+T_{6+r}-$ $\lambda^{(r)}$. Indeed,

$$
\begin{aligned}
& T_{19}-10 T_{13}+T_{7}+12=35890-10(927)+24+12 \\
& =28(952)=28 s_{2}^{(6,1)}, \\
& T_{20}-10 T_{14}+T_{14}-6=66012-10(1705)+44-6 \\
& =28(1750)=28 s_{2}^{(6,2)}, \\
& \vdots \\
& T_{24}-10 T_{18}+T_{18}-10=755476-10(19513)+504 \\
& -10=28 s_{2}^{(6,6)} .
\end{aligned}
$$

So if we assume $28 s_{t-1}^{(6, r)}=T_{6 t+r}-10 T_{6(t-1)+r}+T_{6(t-2)+r}-\lambda^{(r)}$ then

$$
\begin{aligned}
28 s_{t}^{(6, r)}= & \left(T_{6 t+r}-10 T_{6(t-1)+r}+T_{6(t-2)+r}-\lambda^{(r)}\right) \\
& +28 T_{6 t+r} \\
= & \left(39 T_{6 t+r}-11 T_{6(t-1)+r}+T_{6(t-2)+r}\right)-10 T_{6 t+r} \\
& +T_{6(t-1)+r}-\lambda^{(r)} \\
= & T_{6(t+1)+r}-10 T_{6 t+r}+T_{6(t-1)+r}-\lambda^{(r)} .
\end{aligned}
$$

\section{The Tails $\lambda^{(k, r)}$ of the Partial Sum $s_{t}^{(k, r)}$}

With the use of coefficients $\left(a_{k}, b_{k}\right)$ satisfying the $k$-step Tribonacci rule $T_{k t+r}=a_{k} T_{k(t-1)+r}+b_{k} T_{k(t-2)+r}+T_{k(t-3)+r}$ in Lemma 1, the identities in Theorem 3 can be restated such that

$$
\begin{array}{r}
s_{t}^{(5, r)}=a_{5} s_{t-1}^{(5, r)}+b_{5} s_{t-2}^{(5, r)}+s_{t-3}^{(5, r)}+\lambda^{(r)} \\
\text { with }\left\{\lambda^{(r)}\right\}_{r=1}^{5}=\{-7,3,5,1,9\}, \\
s_{t}^{(6, r)}=a_{6} s_{t-1}^{(6, r)}+b_{6} s_{t-2}^{(6, r)}+s_{t-3}^{(6, r)}+\lambda^{(r)} \\
\text { with }\left\{\lambda^{(r)}\right\}_{r=1}^{6}=\{-12,6,4,-2,8,10\} .
\end{array}
$$

In this sense, we are able to recast Theorem 2 as

$$
\begin{array}{r}
s_{t}^{(3, r)}=a_{3} s_{t-1}^{(3, r)}+b_{3} s_{t-2}^{(3, r)}+s_{t-3}^{(3, r)}+\lambda^{(r)} \\
\text { with }\left\{\lambda^{(r)}\right\}_{r=1}^{3}=\{-1,1,1\}, \\
s_{t}^{(4, r)}=a_{4} s_{t-1}^{(4, r)}+b_{4} s_{t-2}^{(4, r)}+s_{t-3}^{(4, r)}+\lambda^{(r)} \\
\text { with }\left\{\lambda^{(r)}\right\}_{r=1}^{4}=\{-4,4,4,4\} .
\end{array}
$$

We will call $\left\{\lambda^{(r)}\right\}_{r=1}^{k}$ the tail set of $s_{t}^{(k, r)}=\sum_{i=0}^{t} T_{k i+r}$.

Theorem 4. With $\left(a_{k}, b_{k}\right)(1 \leq k \leq 10)$ in Lemma 1 , one has the following:
(1) $s_{t}^{(k, r)}=a_{k} s_{t-1}^{(k, r)}+b_{k} s_{t-2}^{(k, r)}+s_{t-3}^{(k, r)}+\lambda^{(k, r)}$,

(2) $s_{t}^{(k, r)}=\left(1 /\left(a_{k}+b_{k}\right)\right)\left(T_{k(t+1)+r}+\left(b_{k}+1\right) T_{k t+r}+T_{k(t-1)+r}-\right.$ $\left.\lambda^{(k, r)}\right)$,

where the tails $\left\{\lambda^{(k, r)}\right\}$ are defined as follows:

\begin{tabular}{cc}
$k$ & $\left\{\lambda^{(k, r)}\right\}_{r=1}^{k}$ \\
\hline 1 & $\{1\}$ \\
2 & $\{0,2\}$ \\
3 & $\{-1,1,1\}$ \\
4 & $\{-4,4,4,4\}$ \\
5 & $\{-7,3,5,1,9\}$ \\
6 & $\{-12,6,4,-2,8,10\}$ \\
7 & $\{-29,13,9,-7,15,17,25\}$ \\
8 & $\{-48,16,16,-16,16,16,16,48\}$ \\
9 & $\{-87,35,21,-31,25,15,9,49,73\}$ \\
10 & $\{-176,66,44,-66,44,22,0,66,88,154\}$
\end{tabular}

Proof. When $3 \leq k \leq 6$, (1) is due to Theorems 2 and 3. If $k=1$ then $s_{t}^{(1,1)}=\sum_{i=0}^{t} T_{i+1}$ is the sum of all $t+1$ numbers from $T_{1}$ to $T_{t+1}$; hence

$$
\begin{aligned}
s_{1}^{(1,1)}+s_{2}^{(1,1)}+s_{3}^{(1,1)}= & \left(T_{1}+T_{2}\right)+\left(T_{1}+T_{2}+T_{3}\right) \\
& +\left(T_{1}+T_{2}+T_{3}+T_{4}\right) \\
= & \left(T_{1}+T_{2}\right)+T_{3}+T_{4}+T_{5}+T_{1} \\
& -T_{3}=s_{4}^{(1,1)}-1 .
\end{aligned}
$$

So for any $t>0$, the identity $s_{t}^{(1,1)}=s_{t-1}^{(1,1)}+s_{t-2}^{(1,1)}+s_{t-3}^{(1,1)}+\lambda^{(1,1)}$ with $\lambda^{(1,1)}=1$ can be proved by induction.

If $k=2$, making use of the table of $s_{t}^{(2, r)}$, it is easy to see that

$$
s_{3}^{(2,1)}=34=3 s_{2}^{(2,1)}+s_{1}^{(2,1)}+s_{0}^{(2,1)}+\lambda^{(2,1)}
$$

with $\lambda^{(2,1)}=0$,

$s_{3}^{(2,2)}=62=3 s_{2}^{(2,2)}+s_{1}^{(2,2)}+s_{0}^{(2,2)}+\lambda^{(2,2)}$

with $\lambda^{(2,2)}=2$. 
Thus it can be generalized to $s_{t}^{(2, r)}=a_{2} s_{t-1}^{(2, r)}+b_{2} s_{t-2}^{(2, r)}+s_{t-3}^{(2, r)}+$ $\lambda^{(k, r)}$ for any $t>0$ and $1 \leq r \leq 2$, since $\left(a_{2}, b_{2}\right)=(3,1)$.

Now when $7 \leq k \leq 10$, (1) can be observed. For instance, the table of $s_{t}^{(7, r)}$ shows that

$$
\begin{aligned}
& s_{3}^{(7,1)}=71(3181)+15(45)+1-29=71 s_{2}^{(7,1)} \\
& \quad+15 s_{1}^{(7,1)}+s_{0}^{(7,1)}-29, \\
& s_{3}^{(7,2)}=71(5850)+15(82)+1+13=71 s_{2}^{(7,2)} \\
& \quad+15 s_{1}^{(7,2)}+s_{0}^{(7,2)}+13, \\
& \vdots \\
& s_{3}^{(7,7)}=71(123144)+15(1729)+24+25=71 s_{2}^{(7,7)} \\
& \quad+15 s_{1}^{(7,7)}+s_{0}^{(7,7)}+25 .
\end{aligned}
$$

These observations together with mathematical induction imply

$$
\begin{aligned}
s_{t}^{(7, r)} & =71 s_{t-1}^{(7, r)}+15 s_{t-2}^{(7, r)}+s_{t-3}^{(7, r)}+\lambda^{(7, r)} \\
& =a_{7} s_{t-1}^{(7, r)}+b_{7} s_{t-2}^{(7, r)}+s_{t-3}^{(7, r)}+\lambda^{(7, r)} .
\end{aligned}
$$

The rest follows similarly.

Since the coefficients $a_{k}$ and $b_{k}$ satisfy $a_{k}=3 T_{k}-T_{k-6}$ and $b_{k}=-a_{-k}$ in Lemma $1, s_{t}^{(k, r)}$ can be expressed by $T_{k t+r}$, $T_{k(t-1)+r}$, and $T_{k(t-2)+r}$ with $\lambda^{(k, r)}$ such that

$$
\begin{aligned}
& \left(a_{1}+b_{1}\right) s_{t}^{(1, r)}=2 s_{t}^{(1, r)}=T_{(t+1)+r}+2 T_{t+r}+T_{(t-1)+r} \\
& \quad-\lambda^{(1, r)}, \\
& \left(a_{2}+b_{2}\right) s_{t}^{(2, r)}=4 s_{t}^{(2, r)}=T_{2(t+1)+r}+2 T_{2 t+r}+T_{2(t-1)+r} \\
& \quad-\lambda^{(2, r)}, \\
& \quad \vdots \\
& \left(a_{10}+b_{10}\right) s_{t}^{(10, r)}=484 s_{t}^{(10, r)}=T_{10(t+1)+r}+42 T_{10 t+r} \\
& +T_{10(t-1)+r}-\lambda^{(10, r)} .
\end{aligned}
$$

So (2) also follows immediately.

In particular if $k=1$ then $r=1$ and $a_{1}=b_{1}=\lambda^{(1,1)}=1$; Theorem 4 shows

$$
s_{t}^{(1,1)}=s_{t-1}^{(1,1)}+s_{t-2}^{(1,1)}+s_{t-3}^{(1,1)}+1 .
$$

But since $s_{t}^{(1,1)}=\sum_{i=0}^{t+1} T_{i}=S_{t+1}$, we have $S_{t+1}=S_{t}+S_{t-1}+$ $S_{t-2}+1$; this is Lemma 1 in [6]. Moreover Theorem 4 implies

$$
\begin{aligned}
s_{t}^{(1,1)} & =\frac{1}{2}\left(T_{t+2}+2 T_{t+1}+T_{t}-1\right) \\
& =\frac{1}{2}\left(T_{t+3}+T_{t+1}-1\right) .
\end{aligned}
$$

It means $S_{t+1}=(1 / 2)\left(T_{t+3}+T_{t+1}-1\right)$, that is, Theorem 2 in [6].

\section{Cyclic Rule for Partial Sums}

The partial sum $s_{t}^{(k, r)}$ and its tail $\lambda^{(k, r)}$ were discussed when $1 \leq k \leq 10$. We investigate them for all $k>0$ by showing certain cyclic rules.

Theorem 5. For any $k>0, s_{t}^{(k, r)}$ satisfies the following cyclic rules:

(1) $s_{t}^{(k, r)}+s_{t}^{(k, r+1)}+s_{t}^{(k, r+2)}=s_{t}^{(k, r+3)}$ for all $1 \leq r \leq k-3$;

(2) $s_{t}^{(k, k-2)}+s_{t}^{(k, k-1)}+s_{t}^{(k, k)}=s_{t+1}^{(k, 1)}-1$;

(3) $s_{t}^{(k, k-1)}+s_{t}^{(k, k)}+s_{t+1}^{(k, 1)}=s_{t+1}^{(k, 2)}$, and $s_{t}^{(k, k)}+s_{t+1}^{(k, 1)}+s_{t+1}^{(k, 2)}=$ $s_{t+1}^{(k, 3)}$.

Proof. When $1 \leq r \leq k-3$, (1) is clear from

$$
\begin{aligned}
s_{t}^{(k, r)} & +s_{t}^{(k, r+1)}+s_{t}^{(k, r+2)} \\
= & \sum_{i=0}^{t}\left(T_{k i+r}+T_{k i+(r+1)}+T_{k i+(r+2)}\right)=\sum_{i=0}^{t} T_{k i+(r+3)} \\
= & s_{t}^{(k, r+3)} .
\end{aligned}
$$

And (2) also follows from

$$
\begin{aligned}
s_{t}^{(k, k-2)}+s_{t}^{(k, k-1)}+s_{t}^{(k, k)} & \\
= & \sum_{i=0}^{t}\left(T_{k i+(k-2)}+T_{k i+(k-1)}+T_{k i+k}\right)=\sum_{i=0}^{t} T_{k i+(k+1)} \\
= & \sum_{j=1}^{t+1} T_{k j+1}=\sum_{j=0}^{t+1} T_{k j+1}-T_{1}=s_{t+1}^{(k, 1)}-1,
\end{aligned}
$$

since $T_{1}=1$. Moreover

$$
\begin{aligned}
s_{t}^{(k, k-1)}+s_{t}^{(k, k)}+s_{t+1}^{(k, 1)}= & \sum_{i=0}^{t} T_{k i+(k-1)}+\sum_{i=0}^{t} T_{k i+k} \\
& +\sum_{i=0}^{t+1} T_{k i+1} \\
= & \sum_{i=1}^{t+1}\left(T_{k i-1}+T_{k i}+T_{k i+1}\right)+T_{1} \\
= & \sum_{i=1}^{t+1} T_{k i+2}+T_{2}=s_{t+1}^{(k, 2)},
\end{aligned}
$$


because $T_{1}=T_{2}$. Finally, we also have

$$
\begin{aligned}
s_{t}^{(k, k)} & +s_{t+1}^{(k, 1)}+s_{t+1}^{(k, 2)} \\
= & \sum_{i=1}^{t+1} T_{k i}+\sum_{i=1}^{t+1} T_{k i+1}+T_{1}+\sum_{i=1}^{t+1} T_{k i+2}+T_{2} \\
= & \sum_{i=1}^{t+1}\left(T_{k i}+T_{k i+1}+T_{k i+2}\right)+T_{1}+T_{2} \\
= & \sum_{i=1}^{t+1} T_{k i+3}+\left(T_{0}+T_{1}+T_{2}\right)=s_{t+1}^{(k, 3)} .
\end{aligned}
$$

Next theorem is about the cyclic rule of the tail $\left\{\lambda^{(k, r)}\right\}_{r=1}^{k}$ satisfying $s_{t}^{(k, r)}=a_{k} s_{t-1}^{(k, r)}+b_{k} s_{t-2}^{(k, r)}+s_{t-3}^{(k, r)}+\lambda^{(k, r)}$.

Theorem 6. For any $k>0,\left\{\lambda^{(k, r)}\right\}_{r=1}^{k}$ satisfies the following cyclic rules:

(1) $\lambda^{(k, r)}+\lambda^{(k, r+1)}+\lambda^{(k, r+2)}=\lambda^{(k, r+3)}$ for all $1 \leq r \leq k-3$;

(2) $\lambda^{(k, k-2)}+\lambda^{(k, k-1)}+\lambda^{(k, k)}=\lambda^{(k, 1)}+\left(a_{k}+b_{k}\right)$;

(3) $\lambda^{(k, k-1)}+\lambda^{(k, k)}+\lambda^{(k, 1)}=\lambda^{(k, 2)}$, and $\lambda^{(k, k)}+\lambda^{(k, 1)}+\lambda^{(k, 2)}=$ $\lambda^{(k, 3)}$.

Proof. When $1 \leq k \leq 10$, Theorem 4 shows that $\left\{\lambda^{(k, r)}\right\}$ satisfies (1). For instance, if $k=10$, the set $\left\{\lambda^{(10, r)}\right\}=$ $\{-176,66,44,-66,44,22,0,66,88,154\}$ implies $-176+66+$ $44=-66, \ldots, 22+0+66=88$, and $0+66+88=154$.

Suppose $1 \leq r \leq k-3$. Then Theorem 5 gives rise to

$$
\begin{aligned}
\lambda^{(k, r)} & +\lambda^{(k, r+1)}+\lambda^{(k, r+2)} \\
= & \left(s_{t}^{(k, r)}+s_{t}^{(k, r+1)}+s_{t}^{(k, r+2)}\right) \\
& -a_{k}\left(s_{t-1}^{(k, r)}+s_{t-1}^{(k, r+1)}+s_{t-1}^{(k, r+2)}\right) \\
& -b_{k}\left(s_{t-2}^{(k, r)}+s_{t-2}^{(k, r+1)}+s_{t-2}^{(k, r+2)}\right) \\
& -\left(s_{t-3}^{(k, r)}+s_{t-3}^{(k, r+1)}+s_{t-3}^{(k, r+2)}\right) \\
= & s_{t}^{(k, r+3)}-a_{k} s_{t-1}^{(k, r+3)}-b_{k} s_{t-2}^{(k, r+3)}-s_{t-3}^{(k, r+3)} \\
= & \lambda^{(k, r+3)} .
\end{aligned}
$$

Now, for $(2),\left(a_{k}, b_{k}\right)$ in Lemma 1 and $\lambda^{(k, r)}$ in Theorem 4 show

\begin{tabular}{c|c|c|c}
$k$ & $a_{k}+b_{k}$ & $\lambda^{(k, k-2)}+\lambda^{(k, k-1)}+\lambda^{(k, k)}-\left(a_{k}+b_{k}\right)$ & $\lambda^{(k, 1)}$ \\
\hline 3 & 2 & $-1+1+1-(2)$ & -1 \\
4 & 16 & $4+4+4-(16)$ & -4 \\
5 & 22 & $5+1+9-(22)$ & -7 \\
& & $\vdots$ & \\
10 & 484 & $66+88+154-(484)$ & -176
\end{tabular}

which proves (2) if $1 \leq k \leq 10$. Now for any $k>0$, we have

$$
\begin{aligned}
\lambda^{(k, k-2)}+\lambda^{(k, k-1)}+\lambda^{(k, k)} \\
=s_{t}^{(k, k-2)}+s_{t}^{(k, k-1)}+s_{t}^{(k, k)} \\
\quad-a_{k}\left(s_{t-1}^{(k, k-2)}+s_{t-1}^{(k, k-1)}+s_{t-1}^{(k, k)}\right) \\
\quad-b_{k}\left(s_{t-2}^{(k, k-2)}+s_{t-2}^{(k, k-1)}+s_{t-2}^{(k, k)}\right) \\
\quad-\left(s_{t-3}^{(k, k-2)}+s_{t-3}^{(k, k-1)}+s_{t-3}^{(k, k)}\right) \\
=\left(s_{t+1}^{(k, 1)}-1\right)-a_{k}\left(s_{t}^{(k, 1)}-1\right)-b_{k}\left(s_{t-1}^{(k, 1)}-1\right) \\
\quad-\left(s_{t-2}^{(k, 1)}-1\right) \\
=\left(s_{t+1}^{(k, 1)}-a_{k} s_{t}^{(k, 1)}-b_{k} s_{t-1}^{(k, 1)}-s_{t-2}^{(k, 1)}\right)-1+a_{k}+b_{k} \\
\quad+1=\lambda^{(k, 1)}+\left(a_{k}+b_{k}\right),
\end{aligned}
$$

due to Theorem 5. Similarly (3) also follows from Theorem 5 such that

$$
\begin{aligned}
\lambda^{(k, k-1)}+ & \lambda^{(k, k)}+\lambda^{(k, 1)} \\
= & s_{t}^{(k, k-1)}+s_{t}^{(k, k)}+s_{t}^{(k, 1)} \\
& -a_{k}\left(s_{t-1}^{(k, k-1)}+s_{t-1}^{(k, k)}+s_{t-1}^{(k, 1)}\right) \\
& -b_{k}\left(s_{t-2}^{(k, k-1)}+s_{t-2}^{(k, k)}+s_{t-2}^{(k, 1)}\right) \\
& -\left(s_{t-3}^{(k, k-1)}+s_{t-3}^{(k, k)}+s_{t-3}^{(k, 1)}\right) \\
= & s_{t+1}^{(k, 2)}-a_{k} s_{t}^{(k, 2)}-b_{k} s_{t-1}^{(k, 2)}-s_{t-2}^{(k, 2)}=\lambda^{(k, 2)} .
\end{aligned}
$$

And the rest follows analogously.

The identity $\lambda^{(k, r)}=s_{t}^{(k, r)}-a_{k} s_{t-1}^{(k, r)}-b_{k} s_{t-2}^{(k, r)}-s_{t-3}^{(k, r)}$ implies that $\left\{\lambda^{(k, r)}\right\}$ does not depend on $t$. Hence without loss of generality if we assume $t=3$ then

$$
\lambda^{(k, r)}=s_{3}^{(k, r)}-a_{k} s_{2}^{(k, r)}-b_{k} s_{1}^{(k, r)}-s_{0}^{(k, r)}
$$

But since $s_{0}^{(k, r)}=T_{r}, s_{1}^{(k, r)}=T_{r}+T_{k+r}, s_{2}^{(k, r)}=T_{r}+T_{k+r}+T_{2 k+r}$, and $s_{3}^{(k, r)}=T_{r}+T_{k+r}+T_{2 k+r}+T_{3 k+r}=2 T_{r}+\left(1+b_{k}\right) T_{k+r}+$ $\left(1+a_{k}\right) T_{2 k+r}$, we have

$$
\lambda^{(k, r)}=\left(1-a_{k}-b_{k}\right) T_{r}+\left(1-a_{k}\right) T_{k+r}+T_{2 k+r} .
$$


Therefore we are able to have tails $\left\{\lambda^{(k, r)}\right\}$ for all $k>10$; for instance,

$$
\begin{aligned}
& \left\{\lambda^{(11, r)}\right\}=\{-305,105,81,-119,67,29, \\
& -23,73,79,129,281\}, \\
& \left\{\lambda^{(12, r)}\right\}=\{-564,212,131,-220,124,36, \\
& \quad-60,100,76,116,292,484\}, \\
& \left\{\lambda^{(13, r)}\right\}=\{-1071,387,261,-423,225,63, \\
& \quad-135,153,81,99,333,513,945\},
\end{aligned}
$$

We remark that a special case $s_{t-1}^{(k, k)}=\sum_{i=0}^{t} T_{k i}$ was proved in [5] by making use of the three roots of $x^{3}-x^{2}-x-1=$ 0 . Considering the difficulty of finding roots of the cubic polynomial, the identity $s_{t-1}^{(k, k)}=\left(1 /\left(a_{k}+b_{k}\right)\right)\left(T_{k(t+1)}+\left(b_{k}+\right.\right.$ 1) $\left.T_{k t}+T_{k(t-1)}-\lambda^{(k, k)}\right)$ in Theorem 4 seems a little bit simple and easy.

\section{Conflict of Interests}

The authors declare that there is no conflict of interests regarding the publication of this paper.

\section{References}

[1] K. Kuhapatanakul, "On the sums of reciprocal generalized Fibonacci numbers," Journal of Integer Sequences, vol. 16, no. 7, Article ID 13.7.1, 8 pages, 2013.

[2] H. Ohtsuka and S. Nakamura, "On the sum of reciprocal Fibonacci numbers," The Fibonacci Quarterly, vol. 46, no. 2, pp. 153-159, 2008.

[3] G. J. Zhang, "The infinite sum of reciprocal of the Fibonacci numbers," Journal of Mathematical Research and Exposition, vol. 31, no. 6, pp. 1030-1034, 2011.

[4] J. Feng, "More identities on the Tribonacci numbers," Ars Combinatoria, vol. 100, pp. 73-78, 2011.

[5] N. Irmak and M. Alp, "Tribonacci numbers with indices in arithmetic progression and their sums," Miskolc Mathematical Notes, vol. 14, no. 1, pp. 125-133, 2013.

[6] E. Kilic, "Tribonacci sequences with certain indices and their sums," Ars Combinatoria, vol. 86, pp. 13-22, 2008.

[7] S. Pethe, "Some identities for tribonacci sequences," Fibonacci Quarterly, vol. 26, no. 2, pp. 144-151, 1988.

[8] E. Choi and J. Jo, "Identities involving tribonacci numbers," Journal of the Chungcheong Mathematical Society, vol. 28, no. 1, pp. 39-51, 2015. 


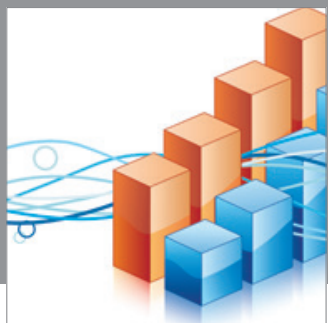

Advances in

Operations Research

mansans

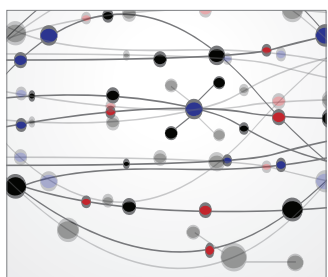

The Scientific World Journal
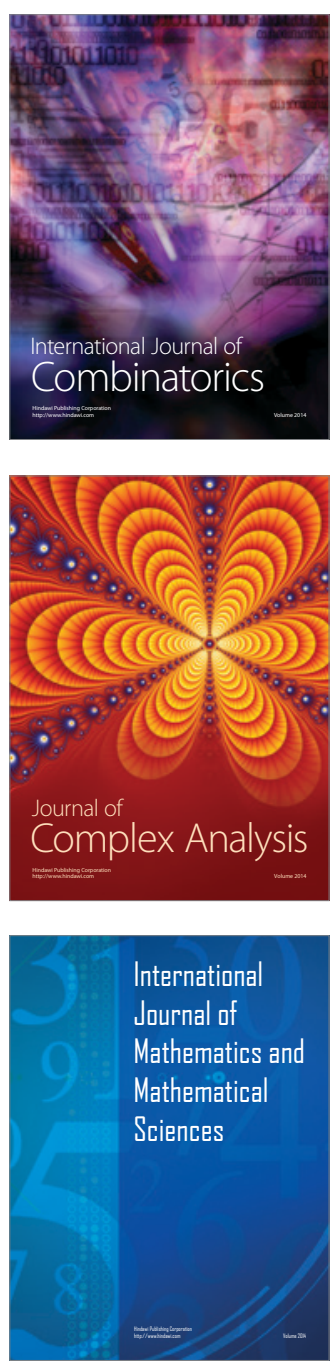
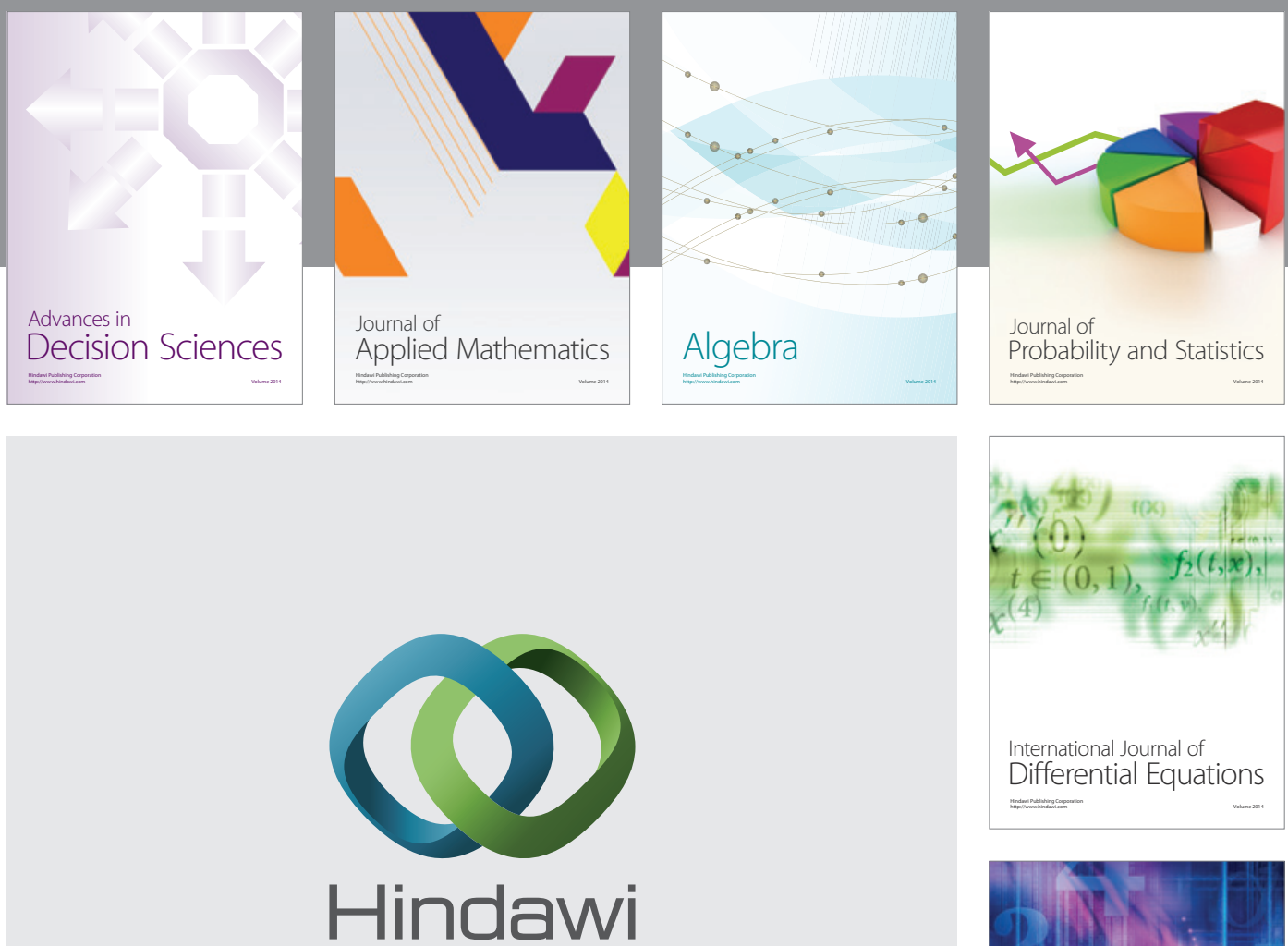

Submit your manuscripts at http://www.hindawi.com
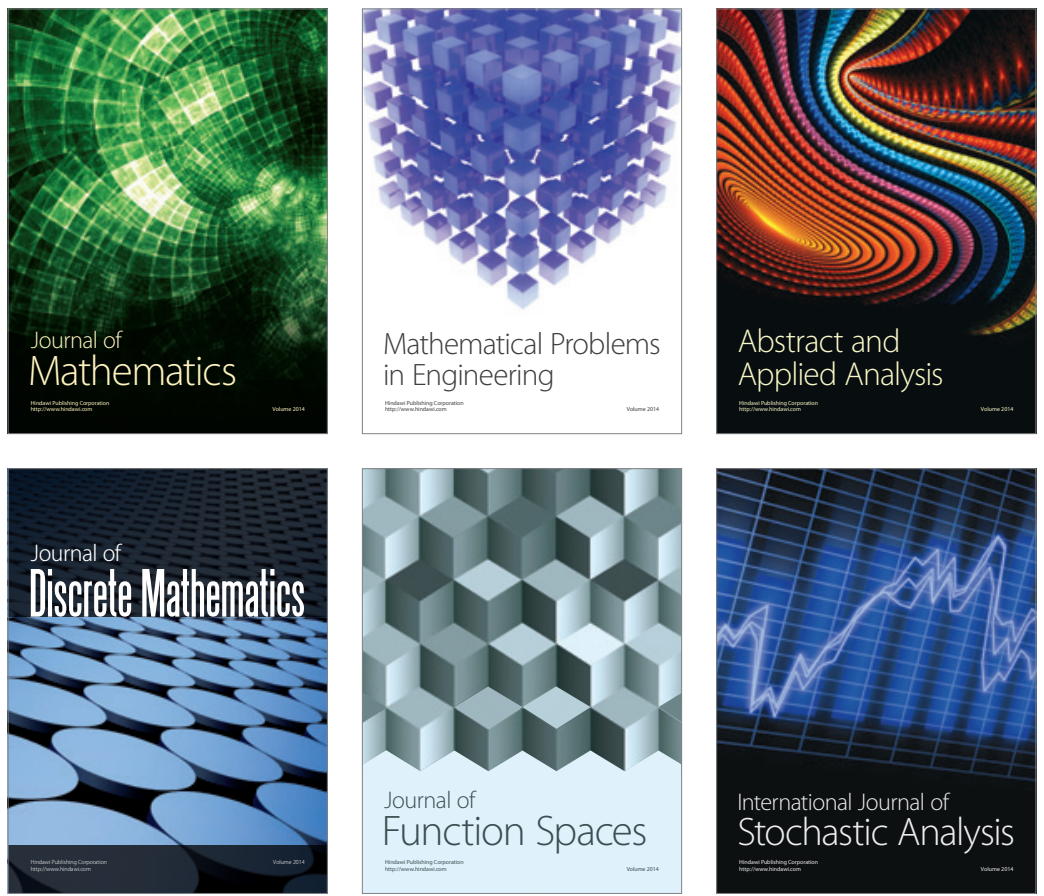

Journal of

Function Spaces

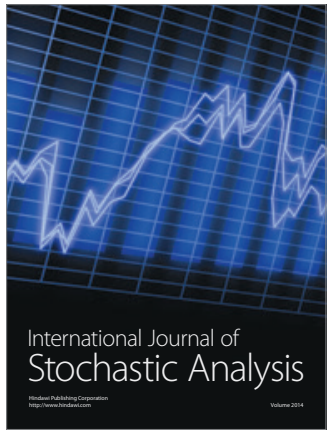

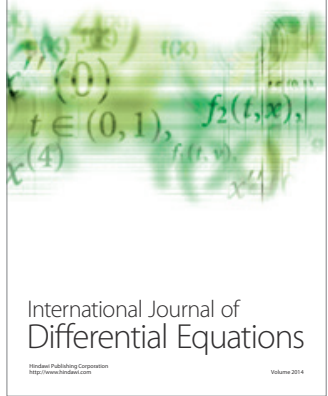
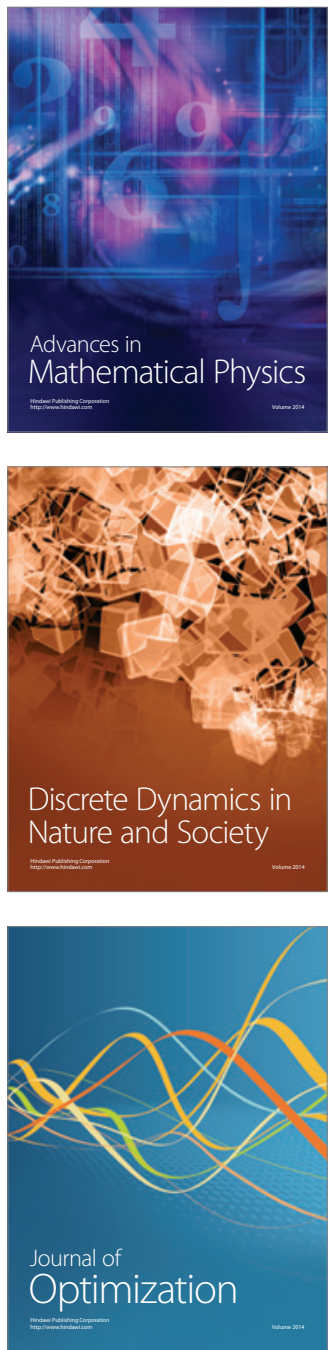